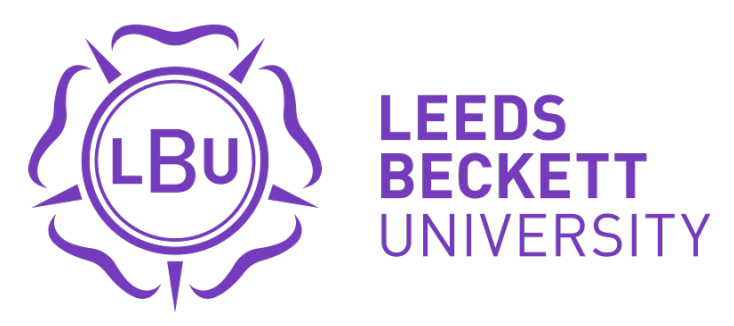

Citation:

Brooks, $\mathrm{R}$ and Bannigan, $\mathrm{K}$ (2018) Occupational therapy interventions in child and adolescent mental health: a mixed methods systematic review protocol. JBI Database of Systematic Reviews and Implementation Reports. ISSN 2202-4433 DOI: https://doi.org/10.11124/JBISRIR-2017-003612

Link to Leeds Beckett Repository record:

https://eprints.leedsbeckett.ac.uk/id/eprint/4915/

Document Version:

Article (Accepted Version)

The aim of the Leeds Beckett Repository is to provide open access to our research, as required by funder policies and permitted by publishers and copyright law.

The Leeds Beckett repository holds a wide range of publications, each of which has been checked for copyright and the relevant embargo period has been applied by the Research Services team.

We operate on a standard take-down policy. If you are the author or publisher of an output and you would like it removed from the repository, please contact us and we will investigate on a case-by-case basis.

Each thesis in the repository has been cleared where necessary by the author for third party copyright. If you would like a thesis to be removed from the repository or believe there is an issue with copyright, please contact us on openaccess@leedsbeckett.ac.uk and we will investigate on a case-by-case basis. 


\section{Occupational therapy interventions in child and adolescent mental health: a mixed methods systematic review protocol.}

\section{Authors}

Rob Brooks ${ }^{1}$ Katrina Bannigan ${ }^{2}$

1. School of Clinical and Applied Sciences, Leeds Beckett University, UK 2. School of Health Professions, Plymouth University, UK.

\section{Background}

The mental health of children and adolescents has been reported to be of world-wide concern. ${ }^{1}$ Relevant epidemiological data is inconsistently reported; in the United Kingdom a study between 1999 and 2004 reported that 1 in 10 children and adolescents aged 5 to 16 years of age were diagnosed with a mental health difficulty, including conduct, emotional, attentional and neurodevelopmental disorders. ${ }^{2}$ More recently, 1 in 8 children and adolescents aged 10 to 15 years of age in the United Kingdom self-reported experiencing symptoms of mental ill-health, as measured by the Strengths and Difficulties Questionnaire. ${ }^{3}$ Similar findings have been reported in Australia where 1 in 7 children and adolescents aged 7 to 17 years of age experienced a mental disorder. ${ }^{4}$ Studies have also reported that children and adolescents experience higher levels of anxiety, depression and behavior problems than they did 30 years ago. ${ }^{5,6}$ These changes have occurred within a shifting social and cultural landscape, which has included increased school attainment, poverty, substance use and changes to family structure. ${ }^{7}$

The mental health needs of children and adolescents have become an important agenda for healthcare services. ${ }^{1}$ Good mental health forms the foundation on which children and adolescents develop resilience and skills to become well-rounded individuals who can cope with the complexities of adult life. There is a positive relationship between some mental health disorders experienced in adolescence and future difficulties in adulthood, ${ }^{8}$ although this is complex and multifactorial. ${ }^{7}$ Current world-wide mental health strategy is emphasizing the need for evidence-based mental health interventions. ${ }^{1}$ Improving mental health interventions is an identified and agreed priority by children and adolescents and their parents $/$ carers $^{9}$ and by healthcare professionals. ${ }^{10}$

Child and adolescent mental health services have been shaped by psychiatry, clinical psychology and psychotherapy; current intervention trends are for 'talking therapies', such as cognitive behavioral therapy and family therapy. ${ }^{11} \mathrm{~A}$ recent report has suggested that the evidence for measuring the success of intervention outcomes with this population is flawed and inconclusive. ${ }^{12}$ Occupational therapists have been part of multidisciplinary teams in child and adolescent mental health services for over sixty years, but it has been reported that they have struggled to carve out a profession-specific 
role. ${ }^{13,14}$ The reasons for this are multifactorial, but include an uncoordinated approach to research, particularly the development of profession-specific interventions. ${ }^{15}$ The complexity of occupational therapy as an intervention has also contributed to difficulties in developing effective interventions. ${ }^{16}$

The outcome of occupational therapy is participation, which the International Classification of Functioning Disability and Health ${ }^{17}$ defines as 'involvement in a life situation'. Occupational therapists translate this as 'participation in everyday occupations' ${ }^{18}$ whereby people participate in and are satisfied with their activities of everyday life. This includes the occupations of self-care (such as getting dressed or eating), productivity (such as going to work or school) and leisure/play (such as playing card games or basketball). ${ }^{19}$

Occupational therapy interventions to address participation can be delineated as occupation-based and/or occupation-focused..$^{20}$ Occupation-based interventions involve the client's participation in a meaningful and purposeful occupation within the context of their everyday life; for example, hiking or baking. Occupation-focused interventions use occupational skills training in the context of occupational performance relevant to the person's goals; for example, the provision of adaptive equipment or the teaching of compensatory strategies during the occupation of dressing. ${ }^{20}$ Interventions that focus on changing a person's underlying body functions or structures are not occupation-based or occupation-focused.

A literature search, including databases of systematic reviews such as JBISRIR and PROSPERO, for similar existing systematic reviews identified one earlier review. ${ }^{21}$ This previous review examined occupation-based and activity-based interventions, within the scope of occupational therapy practice for children and adolescents with mental health difficulties. The review included 124 articles and did find evidence for occupational therapy interventions at a universal, targeted and intensive levels. However, this earlier review differed in three key ways from the review proposed in this protocol. Firstly, the interventions included were much broader than occupation-based or occupation-focused interventions; for example, social-emotional interventions, which are not considered by all to be within the scope of occupational therapy practice. Secondly, the earlier review considered interventions for those without mental illness, those at risk of mental illness, as well as those with identified mental health disorders. Thirdly, the review evaluated outcomes related to psychosocial component skills, such as social interaction and task-focus that were believed to lead to participation in occupations, rather than outcomes being related to occupational participation itself. The review detailed in this protocol will therefore be the first where the population have an identified mental health difficulty, the intervention is occupation-based and/or occupation-focused occupational therapy, and the outcome is related to occupational participation.

Occupational therapy is a complex intervention because it contains several interacting components. ${ }^{16}$ Therefore, to develop an evidence-based occupational therapy intervention it is suggested that a rigorous approach, such as the Medical Research Council (MRC) framework ${ }^{22}$ is utilized. The MRC guidance reports five elements of intervention design: development, piloting, evaluating, reporting and implementing. This review will contribute to addressing the first element: intervention development. Intervention development is further divided into three stages: (i) identifying the evidence base, (ii) developing theory, and (iii) operationalizing the techniques. ${ }^{22}$ This review will focus on the first stage, identifying the evidence, and will contribute to a program of research to rigorously develop an occupation-based and/or occupation-focused complex intervention for children and adolescents with mental health difficulties. 


\section{Review Question}

The question of this review is what is the effectiveness of occupational therapy on participation in everyday occupations in children and adolescents with mental health difficulties? More specifically, the objective is to identify the evidence of occupational therapy interventions that are occupationbased and/or occupation-focused.

The quantitative objective is to identify the effectiveness of occupational therapy on the participation in everyday occupations of children and adolescents with mental health difficulties.

The qualitative objective is to identify the experiences of occupational therapy interventions on the participation of children and adolescents with mental health difficulties in everyday activities.

The textual objective is to identify narrative, text and opinion about occupational therapy interventions on the participation of children and adolescents mental health that have yet to be subjected to empirical analysis.

\section{Inclusion Criteria Mixed Methods}

\section{Participants}

The review will consider studies that include children and adolescents inclusive of the age range 5-16 years who have any of the mental health difficulties commonly experienced by this age group, for example depression, self-harm, generalized anxiety disorder, post-traumatic stress disorder, attention deficit hyperactivity disorder, autism and eating disorders. ${ }^{7}$ Studies which include participants identified as having more than one mental health difficulty, or a comorbid intellectual or physical disability will be included in the review. This age group was selected because if younger children were included the interventions are more likely to target parents, as children are less likely to be able to address the issues themselves, ${ }^{25}$ and adolescents older than this may overlap with adult interventions and services. ${ }^{2}$

\section{Intervention(s)}

This review will consider studies that evaluate occupational therapy interventions, i.e. interventions that are either occupation-based and/or occupation-focused. ${ }^{20}$ Occupation-based interventions include those where the participant is actively engaged in an occupation that has meaning and/or purpose for them; the occupation itself is the therapeutic agent of change. For example, going swimming or taking a shower. Occupation-focused interventions include those where the participant is engaged in the skills needed to participate in everyday life tasks that are relevant to their goals, and the intent of the intervention is enhanced participation in daily life. For example, the teaching of tying shoe laces with the intent to facilitate independence in dressing. Interventions that focus on changing underlying body structures and functions will be excluded.

The quantitative component of the review will consider studies that evaluate the effectiveness of occupation-based and/or occupation-focused interventions; the qualitative component of this review will consider studies that investigate the experiences of these interventions, and the textual 
component of this review will consider publications that describe these occupational therapy interventions.

\section{Comparator(s)}

This review will compare the studies that compare the intervention to any comparator, including no comparator.

\section{Outcomes}

This review will consider studies that include participation in everyday occupations as an outcome. This outcome will be measured by standardized assessment tools or validated self-report measures of: occupational participation, performance or engagement such as the Canadian Occupational Performance Measure; functional ability, such as the School Function Assessment or satisfaction with activities of daily life, such as the Child Occupational Self-Assessment.

\section{Phenomena of Interest}

The experience of children and adolescents with mental health difficulties receiving occupational therapy intervention that is occupation-based and/or occupation-focused.

\section{Context}

This review will include studies conducted in any context.

\section{Study Types}

This review will consider both experimental and quasi-experimental study designs including randomized controlled trials, non-randomized controlled trials, before and after studies and interrupted time-series studies. In addition, analytical observational studies including prospective and retrospective cohort studies, case-control studies and analytical cross-sectional studies will be considered for inclusion. This review will also consider descriptive observational study designs including case series, individual case reports and descriptive cross-sectional studies for inclusion. The textual component of this review will consider publications that describe occupational therapy interventions, such as practice reports and polices. Studies published in English will be included. Studies published since 1927 will be included as this encompasses the time period from the first occupational therapy journals.

\section{Search Strategy}

The search strategy will aim to find both published and unpublished studies. An initial limited search of MEDLINE and CINHAL has been undertaken followed by analysis of the text words contained in the title and abstract, and of the index terms used to describe article. This informed the development of a search strategy which will be tailored for each information source. A full search strategy for MEDLINE 
is detailed in Appendix 1. The reference list of all studies selected for critical appraisal will be screened for additional studies.

The databases to be searched include: AMED via EBSCO, CINAHL Complete via EBSCO, Cochrane Controlled Trials Register, ERIC via EBSCO, PsychINFO via EBSCO, MEDLINE via PubMed, and OTSeeker. This will include keywords and index terms drawn from the thesaurus for each of the databases to be included in searching. The reference list of all identified reports and articles will be searched for additional studies; this will include the use of backwards and forwards citation tracking.

The trial registers to be searched include: Cochrane Central Register of Controlled Trials. The search for unpublished studies will include: Conference Proceedings Citation Index via Web of Science; Dissertation Abstracts via ProQuest; Google Scholar; Networked Digital Library of Theses and Dissertations; OpenDOAR and Open Grey. ${ }^{26}$

\section{Study Selection}

Following the search, all identified citations will be collated and uploaded into EndNote ${ }^{27}$ and duplicates removed. Titles and abstracts will then be screened by two independent reviewers for assessment against the inclusion criteria for the review. Studies that may meet the inclusion criteria will be retrieved in full and their details imported into JBI SUMARI. ${ }^{28}$ The full text of selected studies will be retrieved and assessed in detail against the inclusion criteria. Full text studies that do not meet the inclusion criteria will be excluded and reasons for exclusion will be provided in an appendix in the final systematic review report. Included studies will undergo a process of critical appraisal. The results of the search will be reported in full in the final report and presented in a Preferred Reporting Items for Systematic Reviews and Meta-Analyses (PRISMA) ${ }^{29}$ flow diagram. Any disagreements that arise between the reviewers will be resolved through discussion, or with a third reviewer.

\section{Assessment of Methodological Quality}

Selected studies will be critically appraised by two independent reviewers at the study level for methodological quality in the review using the standardized critical appraisal instruments from JBI SUMAR ${ }^{28}$ for the following study types: quantitative, qualitative and textual. Any disagreements that arise will be resolved through discussion, or with a third reviewer. All studies, regardless of their methodological quality, will undergo data extraction and synthesis (where possible).

\section{Data Extraction}

Data will be extracted from papers included in the review using the standardized data extraction tool available in JBI SUMARI ${ }^{28}$ by the primary reviewer. The data extracted will include specific details about the interventions, populations, study methods and outcomes of significance to the review question and specific objectives. Authors of papers will be contacted to request missing or additional data where required. 


\section{Data Synthesis}

The following two stages of data synthesis will be conducted, as per JBI guidance on conducting systematic reviews of this nature. ${ }^{30}$

\section{Stage 1}

Quantitative papers will, where possible, be pooled in statistical meta-analysis using JBI SUMARI. ${ }^{28}$ Effect sizes expressed as a relative risk for cohort studies, odds ratios for case control studies (for categorical data) and weighted mean differences (for continuous data), and their $95 \%$ confidence intervals will be calculated for analysis. It is likely that a random effects model will be used and heterogeneity will be assessed statistically using the standard chi-square test, however selection of the meta-analysis model will be guided by Tufanaru et al. ${ }^{31}$ If statistical pooling is not be possible the findings will be presented in narrative form including tables and figures to aid in data presentation where appropriate.

Qualitative research findings will, where possible, be pooled using JBI SUMARI. ${ }^{28}$ This will involve the aggregation or synthesis of findings to generate a set of statements that represent that aggregation, through assembling the findings (Level 1 findings) rated according to their quality and categorizing these findings on the basis of similarity in meaning (Level 2 findings). These categories are then subjected to a meta-synthesis to produce a single comprehensive set of synthesized findings (Level 3 findings) that can be used as a basis for evidence based practice. Where textual pooling is not possible, the findings will be presented in narrative form.

Textual papers selected for retrieval will be assessed by the primary and secondary reviewer for authenticity prior to inclusion in the review using standardized critical appraisal instruments from JBI SUMARI. ${ }^{28}$ Textual paper findings will be analyzed using the procedure described above for metaaggregation. Any disagreements that arise between the reviewers will be resolved through discussion, or with a third reviewer. Each of the individual syntheses will be reported in line with PRISMA ${ }^{29}$ guidelines.

\section{Stage 2}

The findings of each single-method synthesis included in this review will be aggregated. This will involve the configuration of the findings to generate a set of statements that represent that aggregation. This will be achieved through coding to attribute a thematic description to all quantitative data, assembling all of the resulting themes from quantitative and qualitative and textual syntheses.

\section{Assessing Confidence}

A 'summary of findings' table will be created using GRADEPro GDT ${ }^{32}$ software. The GRADE approach for grading the quality of evidence will be followed. The 'Summary of Findings' table will present the following information where appropriate: absolute risks for treatment and control, estimates of relative risk, and a ranking of the quality of the evidence based on study limitations (risk of bias), indirectness, inconsistency, imprecision and publication bias. 
The following outcomes will be included in the 'Summary of Findings' table: objectives, study design, level of evidence, participants, interventions, outcomes, and results.

\section{Conflicts of Interest}

There are no conflicts of interest.

\section{Acknowledgements}

The reviewers wish to acknowledge funding for this review from the Royal College of Occupational Therapists, the support of the Participation in Everyday Life group at University of Plymouth, and the Young Dynamos Young Persons Advisory Research Group in Bradford.

\section{References}

1. World Health Organisation. Comprehensive mental health action plan 2013-2010.2013. Available from: www.who.int/mental health/maternal-child/child adolescent/en/ [Accessed June, 2017)

2. Green H, McGinnity A, Meltzer H, Ford T, Goodman R. Mental health of children and young people in Great Britain, 2004. 2005. Available from: http://content.digital.nhs.uk/catalogue/PUB06116/ment-heal-chil-youn-peop-gb-2004rep2.pdf [Accessed June, 2017].

3. Office of National Statistics. Insights into children's mental health and well-being. 2015. Available from: http://webarchive.nationalarchives.gov.uk/20160105160709/http://www.ons.gov.uk/ons/d cp171766 420239.pdf [Accessed June, 2017]

4. Lawrence D, Johnson S, Hafekost J, Boterhoven De Haan K, Sawyer M, Ainley J, Zubrick SR. The mental health of children and adolescents. Report on the second Australian child and adolescent survey of mental health and wellbeing. Canberra: Department of Health; 2015. Available www.health.gov.au/internet/main/publishing.nsf/Content/9DA8CA21306FE6EDCA257E2700 016945/\$File/child2.pdf [Accessed June, 2017].

5. Collishaw S, Maughan B, Natarajan L, Pickles A. Trends in adolescent emotional problems in England: a comparison of two national cohorts twenty years apart. JCPP 2010;51:885-894.

6. Maughan B, Collishaw S, Meltzer H, Goodman R. Recent trends in UK child and adolescent mental health. Soc Psychiatry and Psychiatr Epidemiol 2008;43:305-310.

7. Collishaw S. Annual research review: secular trends in child and adolescent mental health. JCPP. 2015:56(3);370-393.

8. Patton GC, Coffey C, Romaniuk H, Mackinnon P, Carlin JB, Degenhardt L, Olsson CA, Moran P. The prognosis of common mental health disorders in adolescents: a 14-year prospective cohort study. The Lancet 2014;383(9926):1404-1411.

9. Morris C, Simkiss D, Busk M, Morris M, Allard A, Denness J, Janssens A, Stimson A, Coghill J, Robinson $\mathrm{K}$, Fenton $\mathrm{M}$, Cowan $\mathrm{K}$. Setting research priorities to improve the health of children and young people with neurodisability: A British Academy of Childhood DisabilityJames Lind Alliance Research Priority Setting Partnership. BMJ Open 2015;5:e006233.

10. Janssens A, Williams J, Tomlinson R, Logan S, Morris C. Health outcomes for children with neurodisability: what do professional regard as primary targets? ADC 2014;99:927-932.

11. Cottrell D, Kraam A. Growing up? A history of Child and Adolescent Mental Health Services (1987-2005). Children and Young People's Mental Health 2005;10(3):111-117. 
12. Wolpert M, Jacob J, Napoleone E, Whale A, Calderon A, Edbrooke-Childs J. Child- and Parentreported Outcomes and Experience from Child and Young People's Mental Health Services 2011-2015. London: CAMHS Press; 2016.

13. Harrison $M$, Forsyth $K$. Developing a vision for therapists working within children and young people's mental health services: Poised or paused? BJOT 2005;68(4):181-185.

14. Hardaker L, Halcomb EJ, Griffiths R, Bolzan N, Arblaster K. The role of the occupational therapist in adolescent mental health: A critical review of the literature. AeJAMH 2007;6(3):110.

15. Bendixen RM, Kreider CM. Centennial vision - Review of occupational therapy research in the practice area of children and youth. AJOT 2011;65(3): 351-359.

16. Creek J. Occupational therapy defined as a complex intervention. London: RCOT 2003.

17. World Health Organisation International classification of functioning, disability and health (ICF). 2015. Available from www.who.int/classifications/icf/en/ [Accessed June, 2017].

18. World Federation of Occupational Therapists. Definitions of occupational therapy from member organisation. 2013. Available from www.wfot.org/ResourceCentre.aspx [Accessed June, 2017].

19. Polatajko HJ, Davis J, Stewart D, Cantin N, Amoroso B, Purdie L, Zimmerman D. Specifying the domain of concern: Occupation as core. In: Townsend E, Polatajko HJ, editors. Enabling occupation II: Advancing an occupational therapy vision for health, well-being, and justice through occupation, $2^{\text {nd }}$ edition. Ottawa: CAOT Publishing, 2013;13-36.

20. Fisher A. Occupation-centred, occupation-based, occupation-focused: Same, same or different? Scand J Occ Ther 2013;20(3):162-173.

21. Arbesman M. Bazyk S, Nochajski, S. Systematic reviews of occupational therapy and mental health promotion, prevention, and intervention for children and youth. AOTA 2013;67(6);120130.

22. Craig P, Dieppe $P$, Macintyre S, Michie S, Nazareth I, Petticrew M, Developing and evaluating complex interventions: the new Medical Research Council guidance. BMJ 2008;337:a1655.

23. Case-Smith J, Weaver LL, Fristad MA. A systematic review of sensory processing interventions for children with autism spectrum disorders. Autism. 2015;19(2):133-148.

24. Lang R, O'Reilly M, Healy O, Rispoli M, Lydon H, Streusand W, Davis T, Kang S, Sigafoos J, Lancioni $G$, Didden R, Giesbers S. Sensory integration therapy for autism spectrum disorders: A systematic review. RASD 2012;6(3):1004-1018.

25. Stewart-Brown SL, Schrader-McMillan A. Parenting for mental health: what does the evidence say we need to do? Report of Work package 2 of the DataPrev project. Health Promot Int 2011;26:supplement 1.

26. Public Health England. Index of grey literature and alternative sources and resources. Available from: http://phe.baileysolutions.co.uk/custompage6.aspx [Accessed, June 2017).

27. Clarivate Analytics. EndNote X8.2 2017. [Computer software] Available from: http://endnote.com/product-details [Accessed February, 2018].

28. The Joanna Briggs Institute. SUMARI. Available from: www.jbisumari.org/ [Accessed February, 2018].

29. Moher, D, Liberati, A, Tetzlaff, J, Altman, DG. Preferred reporting items for systematic reviews and meta-analyses: the PRISMA statement. PLoS medicine 2009;6:e1000097.

30. Pearson A, White H, Bath-Hextall F, Apostolo J, Salmond S, Kirkpatrick P. Methodology for JBI mixed methods systematic review. The Joanna Briggs Institute reviewers' manual. South Australia: JBI; 2014;5-34.

31. Tufanaru C, Munn Z, Stephenson M, Aromataris E. Fixed or random effects meta-analysis? Common methodological issues in systematic reviews of effectiveness. Int J Evid Based Healthc. 2015; 13:196-207. 
32. GRADEpro GDT: GRADEpro Guideline Development Tool [Software]. McMaster University, 2015 (developed by Evidence Prime, Inc.). Available from: gradepro.org [Accessed February, 2018)

\section{Appendix 1 - Search Strategy}

Example draft MEDLINE via PubMed search. Search conducted by R Brooks on $4^{\text {th }}$ August 2017

Term

1. child [MeSH Term]

2. child* [Title/Abstract]

3. adolescent [MeSH Term]

4. young person [Title/Abstract]

5. young people [Title/Abstract]

6. juvenile* [Title/Abstract]

7. boy [Title/Abstract]

8. boys [Title/Abstract]

9. girl [Title/Abstract]

10. girls [Title/Abstract]

11. kid [Title/Abstract]

12. kids [Title/Abstract]

13. 1 OR 2 OR 3 OR 4 OR 5 OR 6 OR 7 OR 8 OR 9 OR 10 OR 11 OR 12

14. mental health [MeSH Term]

15. mental disorders [MeSH Term]

16. mentally ill persons [MeSH Term]

17. psychiatric illness [Title/Abstract]

18. psychiatric disorders [Title/Abstract]

19. behavior, self-injurious [MeSH Term]

20. mental health services [MeSH Term]

21. adolescent health [MeSH Term]

22. child health [MeSH Term]

23. 13 OR 14 OR 15 OR 16 OR 17 OR 18 OR 19 OR 20 OR21 OR 22

24. occupational therapy [MeSH Term]

25. occupational therapists [MeSH Term]

26. occupation-based [Title/Abstract]
Hits

1717863

1227516

1798014

839

21402

64770

52728

51029

79179

1567

4941

3123620

28453

1079045

5717

5836

7947

61047

84482

365

799

1190116

11767

42

242 
27. occupation-focused [Title/Abstract]

35

28. occupation-cent* [Title/Abstract] 32

29. 24 OR 25 OR 26 OR 27 OR 28 11946

30. activities of daily living [MeSH Term] 59641

31. activities of daily life [Title/Abstract]

876

32. everyday life [Title/Abstract]

7000

33. everyday activities [Title/Abstract]

1626

34. life skills [Title/Abstract]

874

35. education [MeSH Term]

691142

36. work [MeSH Term]

26314

37. employment [MeSH Term]

74427

38. leisure activities [MeSH Term]

199447

39. sleep [MeSH Term]

68771

40. out of school activities [Title/Abstract]

45

41. Satisfaction [Title/Abstract]

104886

42. Performance [Title/Abstract]

43. Participation [Title/Abstract]

44. Function* [Title/Abstract]

45. 30 OR 31 OR 32 OR 33 OR 34 OR 35 OR 36 OR 37 OR 38 OR 39 OR 40

OR 41 OR 42 OR 43 OR 44

4710596

13 AND 23 AND 29 AND 45
443 